\title{
A joint OFDM PAPR reduction and data decoding scheme with no SI estimation

\author{
Saheed A. Adegbite* ${ }^{*}$, Scott G. McMeekin and Brian G. Stewart ${ }^{\dagger}$
}

\begin{abstract}
The need for side information (SI) estimation poses a major challenge when selected mapping (SLM) is implemented to reduce peak-to-average power ratio (PAPR) in orthogonal frequency division multiplexing (OFDM) systems. Recent studies on pilot-assisted SI estimation procedures suggest that it is possible to determine the SI without the need for SI transmission. However, SI estimation adds to computational complexity and implementation challenges of practical SLM-OFDM receivers. To address these technical issues, this paper presents the use of a pilot-assisted cluster-based phase modulation and demodulation procedure called embedded coded modulation (ECM). The ECM technique uses a slightly modified SLM approach to reduce PAPR and to enable data recovery with no SI transmission and no SI estimation. In the presence of some non-linear amplifier distortion, it is shown that the ECM method achieves similar data decoding performance as conventional SLM-OFDM receiver that assumed a perfectly known SI and when the SI is estimated using a frequency-domain correlation approach. However, when the number of OFDM subcarriers is small and due to the clustering in ECM, the modified SLM produces a smaller PAPR reduction gain compared with conventional SLM.
\end{abstract}

Keywords: Low computational complexity, Orthogonal frequency division multiplexing (OFDM), Peak-to-average power ratio (PAPR), Selective mapping (SLM), Side information (SI) estimation

\section{Introduction}

Orthogonal frequency division multiplexing (OFDM) is adopted in many high-speed wireless communication systems including long-term evolution (LTE) because it offers high data rate transmission, immunity to multipath fading and high spectral efficiency [1-3]. However, OFDM produces signals with high peak-to-average power ratio (PAPR) due to the coherent summation of subcarrier phases at some time instants [4]. The presence of high PAPR signals causes transmitter front-end high power amplifiers (HPAs) to operate in their non-linear region, thereby causing signal distortion and bit error rate (BER) degradation [4]. In theory, designing HPAs with a large linear region, i.e. large input back off (IBO) level, can reduce non-linear distortion at the cost of reduced amplifier efficiency. A comprehensive review of common PAPR reduction solutions for OFDM can be found in [4-6].

*Correspondence: sa.adegbite@gmail.com

${ }^{\dagger}$ Equal contributor

School of Engineering and Built Environment, Glasgow Caledonian University, 70 Cowcaddens Road, G4 0BA Glasgow, UK
Amongst these methods, selected mapping (SLM) in [7] offers improved PAPR reduction performance compared to other PAPR reduction methods such as partial transmit sequences (PTS) [8]. Though both SLM and the PTS schemes are both attractive solutions, the SLM technique is considered in this paper because (1) it is more compatible with the presented solution in this paper and (2) according to the study found in [9], the SLM scheme requires relatively reduced computational complexity over the PTS scheme in order to achieve similar level of performance in the form of adjacent channel leakage ratio (ACLR) and BER.

SLM uses a number of phase rotation sequence vectors to produce alternative OFDM signals with different levels of PAPR, from which the modified signal with the lowest PAPR level is selected. As a consequence, the identity of the phase rotation sequence vector that produces this low PAPR signal must be known at the receiver through what is generally known as side information. When SLM is implemented, it is customary for a conventional SLMOFDM receiver to perform some form of side information 
(SI) estimation because this SI contains critical information, which must be determined to achieve successful reception of payload data [10-13]. To achieve SI estimation, it is possible to transmit a channel-coded form of the SI. However, SI transmission in any form reduces data throughput and is unattractive for practical applications [14].

For this reason, studies in [10-13] introduce alternative methods where SLM-OFDM data decoding is achieved through some statistical SI estimation decision procedures without the need for SI transmission. These methods presume that all the candidate phase rotation sequences have a well-defined structure and hence can be either reconstructed at the receiver or retrieved from system's memory. The SI estimation is achieved in [10] using a form of blind maximum-likelihood (ML) detection criterion and in [11] using an extended subcarrier symbol approach. To reduce the level of computations associated with both [10] and [11], Park et al. [12] and Hong et al. [13] use pilot sequences embedded within the transmitted OFDM block for the purpose of SI estimation and subsequently channel estimation. The approach introduced by Park et al. [12] is based on a form of pilot-aided ML (PAML) detection using a slightly modified SLM while the scheme introduced by Hong et al. [13] is based on conventional SLM, and it computes an SI estimate from a frequency-domain correlation (FDC) function. Consequently, the modified SLM used in [12] results in a slightly less PAPR reduction performance compared with conventional SLM. As a consequence, the FDC-based pilotassisted SI estimation (PASIE) scheme is chosen for the purpose of comparison with the method presented in this paper. However, in terms of SI estimation, both Park et al. [12] and Hong et al. [13] produce similar BER performance, assuming perfect synchronisation between the transmitter and the receiver. In summary, the need for SI estimation poses two critical challenges: (1) it adds to the computational complexity of the receiver and (2) it introduces additional implementation constraints because the receiver must know whether to implement or to bypass the SI estimation procedures, i.e. the receiver must know when PAPR reduction is implemented [15].

To address these SI-related practical challenges, this paper investigates the use of a joint pilot-assisted PAPR and data decoding method based on a clustered OFDM approach called embedded coded modulation (ECM). It will be shown that though the PTS PAPR reduction method employs a form of clustered OFDM, the implementation of the ECM technique is different from the PTS scheme as it employs a modified SLM called clustered SLM. The fundamental difference between conventional SLM and ECM is that the latter is a pilot-aided clustered data decoding scheme with a self-channel mitigation feature and requires no SI estimation through the use of pilots that are traditionally associated with channel estimation. To demonstrate ECM's capabilities, its PAPR reduction and BER performance is evaluated and compared with two other methods, i.e. an equivalent conventional SLM-based clustered OFDM approach that assumes (1) perfect SI estimation at the receiver and (2) the use of an FDC-based SI estimation scheme studied in [13].

The structure of the paper is as follows. Section 2 describes a pilot-assisted baseband SLM-OFDM transceiver model used for the investigations in this paper. The basic principles of the SLM approach and the FDC SI estimation methods are also described in this section. Section 3 presents the ECM method and its application to achieve combined PAPR reduction and data recovery through a normalised channel mitigation approach. Section 4 simulates, discusses, and compares both PAPR reduction and BER performance between ECM and conventional SLM-OFDM (with perfect SI estimation and with FDC based SI estimation). Finally, conclusions based on the results are presented in Section 5.

\section{System model}

As a background for understanding the rationale behind ECM, this section provides a brief overview of the application of SLM to a basic pilot-assisted OFDM system. Figure 1 shows a block diagram representation of a pilotassisted SLM-OFDM transceiver. A description of the transmitter and receiver of conventional SLM-OFDM can be summarised as follows.

\subsection{Transmitter}

Consider an OFDM symbol block $\boldsymbol{X}$ of size $N_{v}$. For $0 \leq$ $k \leq N_{v}-1$ where $k$ represent the subcarrier index, $X$ can be represented as

$$
\boldsymbol{X}=\left[\boldsymbol{X}[0] \boldsymbol{X}[1] \boldsymbol{X}[k] \ldots \boldsymbol{X}\left[N_{v}-1\right]\right] .
$$

By letting $X$ be a pilot-assisted OFDM symbol, let $L$ represent the pilot spacing, i.e. the number of subcarriers between two consecutive pilots, then for an equi-spaced pilot structure, the total number of pilots, $N_{p}=N_{v} / L$. Hence, the number of data subcarriers, $N_{d}$ is $N_{v}-N_{p}$.

For an equi-spaced pilot arrangement, a contiguous set of subcarriers within $X$ can be partitioned into a number of clusters as depicted in Fig. 2. Thus, as an example, $X$ can be considered as an aggregate of $N_{c}$ clusters (of the same size) i.e.

$$
\boldsymbol{X}=\left\{\begin{array}{llll}
X_{1} X_{2} X_{c} \ldots X_{N_{c}} & \ldots
\end{array}\right.
$$

where $c$ for $1 \leq c \leq N_{c}$ represents the cluster index and each cluster is denoted by $\boldsymbol{X}_{c}$.

Using, for example, the cluster structure in Fig. 2, $N_{c}=$ $N_{p} / 2$, since each cluster consist of two pilots. Let $W=2 L$ be the cluster size, then for $0 \leq w \leq W-1$ where $w$ is the 

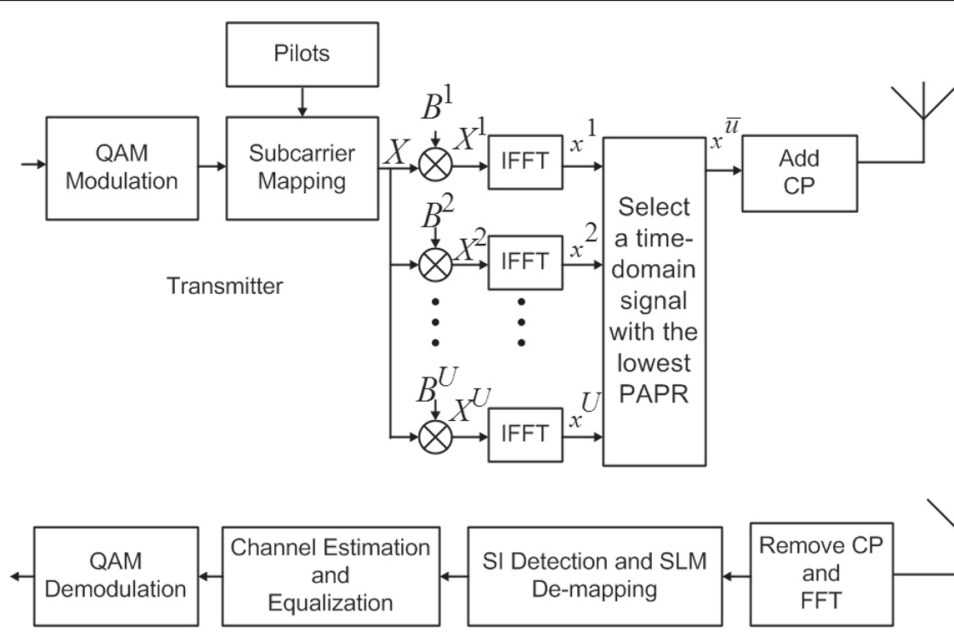

SI Detection and SLM

De-mapping

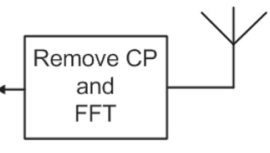

Receiver

Fig. 1 A pilot-assisted conventional SLM-OFDM baseband transmitter-receiver model

cluster subcarrier index, each subcarrier in a given cluster $\boldsymbol{X}_{c}$ is denoted by $\boldsymbol{X}_{c}[w]$. Similar to [16], $\boldsymbol{X}_{c}[w]$ is expressed as

$$
\begin{aligned}
\boldsymbol{X}_{c}[w] & =\boldsymbol{X}[c W+w]=\boldsymbol{X}[k] \\
& =\left\{\begin{array}{l}
\boldsymbol{X}_{c}\left[w_{e}\right]=\boldsymbol{X}\left[c W+w_{e}\right] \\
\boldsymbol{X}_{c}\left[w_{o}\right]=\boldsymbol{X}\left[c W+w_{o}\right] \\
\boldsymbol{X}_{c}\left[w_{d}\right]=\boldsymbol{X}\left[c W+w_{d}\right]
\end{array}\right.
\end{aligned}
$$

where $w_{e}$ and $w_{o}$ are the $w$-indices of the first and second pilots in each cluster, respectively (see Fig. 2). Similarly, $w_{d}$ is associated with data in each cluster. From the expression in (3), it can be seen that both unclustered, $\boldsymbol{X}[k]$ and clustered, $\boldsymbol{X}_{c}[w]$ representations can be used interchangeably.
In polar coordinate form, $\boldsymbol{X}_{c}[w]$ may be expressed as

$$
\boldsymbol{X}_{c}[w]=\boldsymbol{A}_{c}[w] \exp \left(j \theta_{c}[w]\right)
$$

where $\boldsymbol{A}_{c}[w]$ and $\theta_{c}[w]$ are respectively the amplitude and phase components of $\boldsymbol{X}_{c}[w]$.

Using an $N$-point inverse fast Fourier transform (IFFT) where $N>N_{v}$, a time-domain signal $\boldsymbol{x}$ of size $N$ is obtained from $X$. For $0 \leq n \leq N-1$, each time-domain signal sample, $\boldsymbol{x}[n]$ is expressed through [17]

$$
\begin{aligned}
\boldsymbol{x}[n] & =\frac{1}{N} \sum_{k=0}^{N_{v}-1} \boldsymbol{X}[k] \exp (j 2 \pi n k / N) \\
& =\operatorname{IFFT}\{\boldsymbol{X}\}
\end{aligned}
$$

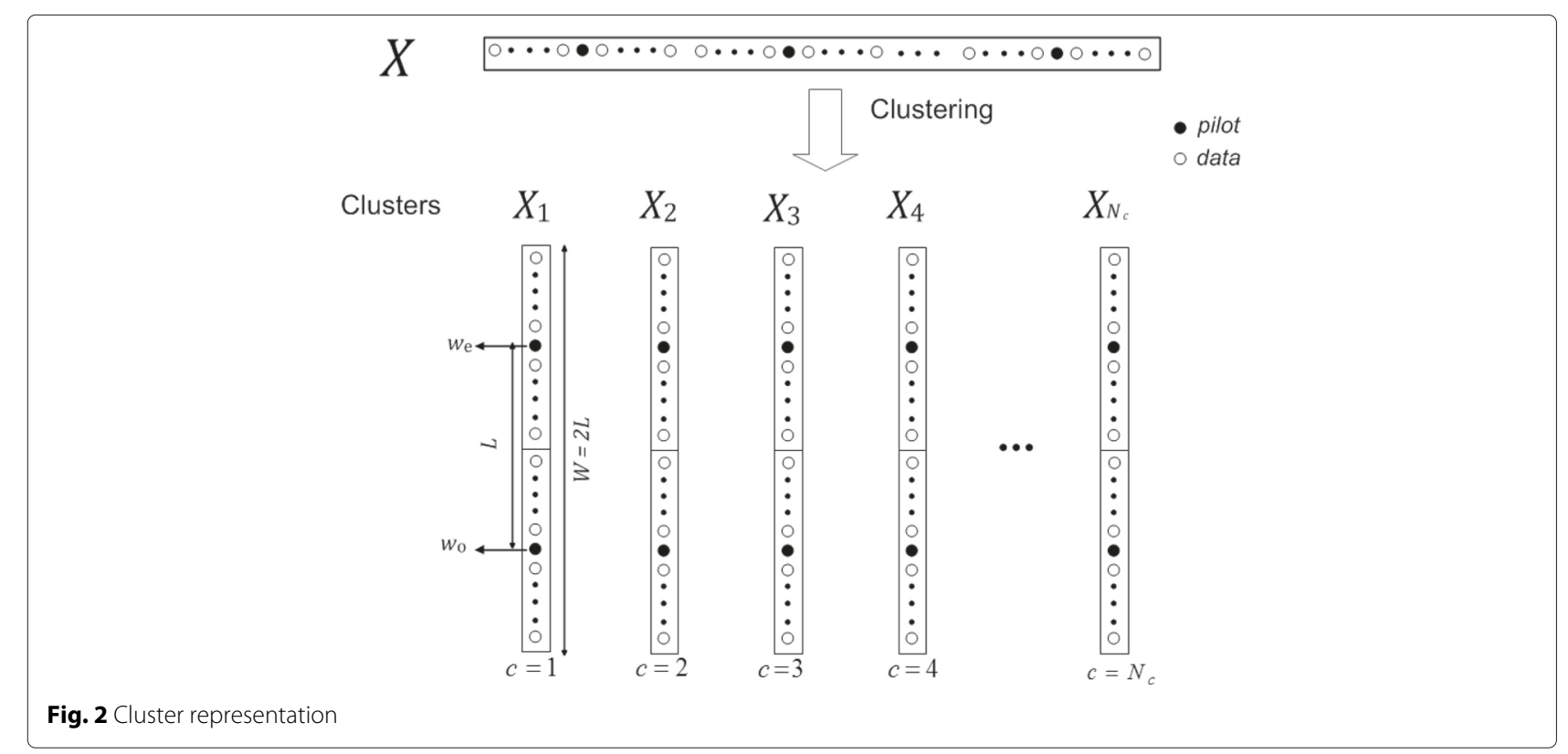


where IFFT $\{\cdot\}$ denotes the IFFT function. Finally, the length of the OFDM signal $x$ is further extended by a cyclic prefix $(\mathrm{CP})$ to mitigate channel fading, reduce inter-symbol interference (ISI) and facilitate the use of frequency-domain equalisation [18]. The PAPR of $\boldsymbol{x}$ is calculated from [19]

$$
\operatorname{PAPR}\{\boldsymbol{x}\}=\frac{\max \left\{|\boldsymbol{x}|^{2}\right\}}{E\left\{|\boldsymbol{x}|^{2}\right\}}
$$

where $E\{\cdot\}$ denotes expectation function. Note that the use of CP has no noticeable influence on the PAPR evaluations [20].

\subsubsection{SLM}

A detailed description of the well-known SLM PAPR reduction technique can be found in [7]. Using $U$ different sequence vectors $\boldsymbol{B}^{u}[k]=\exp \left(j \boldsymbol{\alpha}^{u}[k]\right)$ for $1 \leq u \leq$ $U$ where $\boldsymbol{\alpha}^{u}[k] \in(0, \pi]$ represent positive valued phase sequence values, SLM generates $U$ alternative OFDM signals and selects (for transmission) the modified signal with the lowest PAPR.

Let $\bar{u}$ represent the $u$-index of the phase sequence vector that produced the lowest PAPR signal, $\boldsymbol{x}^{\bar{u}}[n]$ is defined by [13]

$$
\boldsymbol{x}^{\bar{u}}[n]=\operatorname{IFFT}\left\{\boldsymbol{X}[k] \boldsymbol{B}^{\bar{u}}[k]\right\} .
$$

From the expression in (7), it can be noted that in order to achieve successful data recovery, the value of $\boldsymbol{B}^{\bar{u}}[k]$ or its $u$-index $\bar{u}$ must be correctly known or determined at the receiver [21].

\subsection{Receiver}

As in a standard baseband OFDM receiver, all CP samples are first removed from the received signal before transforming the remaining signal samples into the frequency domain through a fast Fourier transform (FFT) to produce $\bar{Y}[k]$, which is given by [13]

$$
\overline{\boldsymbol{Y}}[k]=\boldsymbol{H}[k] \boldsymbol{X}[k] \boldsymbol{B}^{\bar{u}}[k]+\boldsymbol{V}[k] .
$$

The terms $\boldsymbol{H}[k]$ and $\boldsymbol{V}[k]$ respectively represent the channel gain and the independent and additive white Gaussian noise (AWGN) at the $k$ th subcarrier. In a similar manner to $\boldsymbol{X}[k]$ in (3), $\boldsymbol{H}[k]$ and $\boldsymbol{V}[k]$ can also be respectively represented in clustered forms as $\boldsymbol{H}_{c}[w]$ and $\boldsymbol{V}_{c}[w]$. Thus, the expression for $\overline{\boldsymbol{Y}}[k]$ can be re-written as

$$
\overline{\boldsymbol{Y}}_{c}[w]=\boldsymbol{H}_{c}[w] \boldsymbol{X}_{c}[w] \boldsymbol{B}_{c}^{\bar{u}}[w]+\boldsymbol{V}_{c}[w] .
$$

After the FFT, the next stage involves SLM de-mapping of $\bar{Y}[k]$. Normally, at this point, the value of SI must be determined.

\subsubsection{FDC Sl estimation}

Let $\hat{u}$ represent an estimate of the SI. Using the FDC-based SI estimation technique described in [13] and assuming all the $U$ candidate SLM sequences $\boldsymbol{B}^{u}$ are known at the receiver, $\hat{u}$ can be computed from

$$
\hat{u}=\underset{u}{\arg \max } \operatorname{Re}\left\{\boldsymbol{R}^{u}\right\}
$$

where $\boldsymbol{R}^{u}$ is the FDC function, computed from [13]

$$
\boldsymbol{R}^{u}=\frac{1}{N_{p}-1} \sum_{p=1}^{N_{p}-1} \overline{\boldsymbol{H}}^{u}[p] \cdot \overline{\boldsymbol{H}}^{u}[p-1]^{*}
$$

where

$$
\overline{\boldsymbol{H}}^{u}[p]=\left(\overline{\boldsymbol{Y}}[p] \boldsymbol{B}^{u}[p]^{*}\right) / \boldsymbol{X}[p]
$$

and where ${ }^{*}$ is the complex conjugation operator and $p$ for $0 \leq p \leq N_{p}-1$ represents the pilot indices. From the study in [16], the FDC method was shown to require a total of $2 U N_{p}-U$ complex multiplications (CMs) and $U\left(N_{p}-2\right)$ complex additions (CAs).

\subsubsection{SLM de-mapping}

Using the SI estimate $\hat{u}$ and if $\hat{u}=\bar{u}$, a variable denoted by $\boldsymbol{Y}_{c}[w]$ (free of the SLM term $\boldsymbol{B}_{c}^{\bar{u}}[w]$ ) is obtained from

$$
\begin{aligned}
\boldsymbol{Y}_{c}[w] & =\overline{\boldsymbol{Y}}_{c}[w] \boldsymbol{B}_{c}^{\hat{u}}[w]^{*} \\
& =\left\{\begin{array}{l}
\boldsymbol{Y}_{c}\left[w_{e}\right]=\boldsymbol{H}_{c}\left[w_{e}\right] \boldsymbol{X}_{c}\left[w_{e}\right]+\boldsymbol{V}^{\prime}{ }_{c}\left[w_{e}\right], \\
\boldsymbol{Y}_{c}\left[w_{o}\right]=\boldsymbol{H}_{c}\left[w_{o}\right] \boldsymbol{X}_{c}\left[w_{o}\right]+\boldsymbol{V}^{\prime}{ }_{c}\left[w_{o}\right], \\
\boldsymbol{Y}_{c}\left[w_{d}\right]=\boldsymbol{H}_{c}\left[w_{d}\right] \boldsymbol{X}_{c}\left[w_{d}\right]+\boldsymbol{V}^{\prime}{ }_{c}\left[w_{d}\right],
\end{array}\right.
\end{aligned}
$$

where $V^{\prime}{ }_{c}[w]=V_{c}[w] B_{c}^{\hat{u}}[w]^{*}$. After SLM de-mapping, the next stage involves channel estimation and channel equalisation.

\subsubsection{Channel estimation and equalisation}

In practical systems, it is usually assumed that the transmitted pilots are known at the receiver. Using, for example, a least squares (LS) channel estimation method, sub-channel estimates $\hat{\boldsymbol{H}}_{c}\left[w_{e}\right]$ and $\hat{\boldsymbol{H}}_{c}\left[w_{o}\right]$ can be computed through [22]

$$
\begin{aligned}
\hat{\boldsymbol{H}}_{c}\left[w_{e}\right] & =\boldsymbol{Y}_{c}\left[w_{e}\right] / \boldsymbol{X}_{c}\left[w_{e}\right] \\
& =\boldsymbol{H}_{c}\left[w_{e}\right]+\left(\boldsymbol{V}^{\prime}{ }_{c}\left[w_{e}\right] / \boldsymbol{X}_{c}\left[w_{e}\right]\right), \\
\hat{\boldsymbol{H}}_{c}\left[w_{o}\right] & =\boldsymbol{Y}_{c}\left[w_{o}\right] / \boldsymbol{X}_{c}\left[w_{o}\right] \\
& =\boldsymbol{H}_{c}\left[w_{o}\right]+\left(\boldsymbol{V}^{\prime}{ }_{c}\left[w_{o}\right] / \boldsymbol{X}_{c}\left[w_{o}\right]\right) .
\end{aligned}
$$

At high signal-to-noise ratio (SNR), the additive noise terms in (15) become negligible and as a result, the expressions for $\hat{\boldsymbol{H}}_{c}\left[w_{e}\right]$ and $\hat{\boldsymbol{H}}_{c}\left[w_{o}\right]$ are reduced to

$$
\hat{\boldsymbol{H}}_{c}\left[w_{e}\right] \approx \boldsymbol{H}_{c}\left[w_{e}\right] \text { and } \hat{\boldsymbol{H}}_{c}\left[w_{o}\right] \approx \boldsymbol{H}_{c}\left[w_{o}\right] .
$$

Through, for example, a linear interpolation between $\hat{\boldsymbol{H}}_{c}\left[w_{e}\right]$ and $\hat{\boldsymbol{H}}_{c}\left[w_{o}\right]$, data sub-channel estimate $\hat{\boldsymbol{H}}_{c}\left[w_{d}\right]$ is 
obtained. Using the data sub-channel estimate, a channel equalised data term $\hat{\boldsymbol{Y}}_{c}\left[w_{d}\right]$ is obtained from

$$
\begin{aligned}
\hat{\boldsymbol{Y}}_{c}\left[w_{d}\right] & =\boldsymbol{Y}_{c}\left[w_{d}\right] / \hat{\boldsymbol{H}}_{c}\left[w_{d}\right] \\
& \approx \boldsymbol{X}_{c}\left[w_{d}\right] \frac{\boldsymbol{H}_{c}\left[w_{d}\right]}{\hat{\boldsymbol{H}}_{c}\left[w_{d}\right]}+\left(\boldsymbol{V}_{c}{ }_{c}\left[w_{d}\right] / \hat{\boldsymbol{H}}_{c}\left[w_{d}\right]\right) .
\end{aligned}
$$

From the expression in (17), it can be noted at high SNR and in the absence of noise enhancement due to the term $\left(V^{\prime}{ }_{c}\left[w_{d}\right] / \hat{\boldsymbol{H}}_{c}\left[w_{d}\right]\right)$, the use of a standard quadrature amplitude modulation (QAM) demodulation (based on minimum Euclidean distance) produces an estimate of the transmitted data using [16]

$$
\hat{\boldsymbol{X}}_{c}\left[w_{d}\right]=\min _{\mathcal{C}_{q} \in \mathbb{Q}}\left|\hat{\boldsymbol{Y}}_{c}\left[w_{d}\right]-\mathcal{C}_{q}\right|^{2}
$$

where $\mathbb{Q}$ is a set of $Q$ constellation points $\mathcal{C}_{q}$ for $1 \leq q \leq Q$ such that $\hat{\boldsymbol{X}}_{c}\left[w_{d}\right] \in \mathbb{Q}$.

\section{The ECM method}

This section presents the main concepts of the ECM technique in terms of the related PAPR reduction and channel mitigation procedures. In practical systems, pilots are normally interleaved with data to facilitate coherent detection. In ECM, these pilots are employed to enable joint channel mitigation and SI cancellation within a slightly modified SLM-OFDM architecture.

At the transmitter, ECM uses a common modulating phase component, which may be randomly generated to phase-modulate all subcarriers in each cluster, assuming each cluster consists of at least two pilots. This suggests phase modulation on a cluster-to-cluster basis rather than on a subcarrier-to-subcarrier basis as in the case of conventional SLM. Hence, a different modulating phase component can be applied to different clusters. However, in ECM, all subcarriers in a given cluster are modified with the same phase. Therefore, since the pilots are usually known at the receiver, then using a corresponding cluster-based phase demodulation procedure, the applied modulating phase component (on each cluster) can be removed (or demodulated) at the receiver through a simple division process with respect to one of the received pilots without the need to know or determine the value of the modulating phase component.

In a similar manner to conventional SLM, the ECM technique can serve to reduce PAPR. In this case, the applied SLM sequences are designed such that the same phase shift is applied to the contiguous set of subcarriers that forms the cluster. It will be shown later that the main reason for the use of the modified SLM sequences or the clustered SLM sequences (in ECM) is to facilitate channel mitigation and data decoding without the need for SI estimation at the receiver.

\subsection{Transmitter}

For each cluster index $c$, the clustered SLM sequences are defined such that

$$
\boldsymbol{J}_{c}^{u}\left[w_{e}\right]=\boldsymbol{J}_{c}^{u}\left[w_{o}\right]=\boldsymbol{J}_{c}^{u}\left[w_{d}\right]=\boldsymbol{J}_{c}^{u}=\exp \left(j \beta_{c}^{u}\right)
$$

Similar to conventional SLM, the phase sequence vector (amongst all $U$ clustered SLM sequences) that produces the lowest PAPR signal can be identified by $J_{c}^{\bar{u}}=\exp \left(j \beta_{c}^{\bar{u}}\right)$ where $\beta_{c}^{\bar{u}} \in[0, \pi)$ represent the phase value.

Let $\Theta_{c}$ represent the modulating phase component for each cluster, then without loss of generality, it is possible to represent $\Theta_{c}$ as follows:

$$
\Theta_{c}=\beta_{c}^{\bar{u}}
$$

Consider the clustered representation in (3), then using the definition of $\Theta_{c}$ in (20), an ECM-based clustered phase modulation produces $\boldsymbol{E}_{c}[w]$ through

$$
\begin{aligned}
\boldsymbol{E}_{c}[w] & =\boldsymbol{X}_{c}[w] \exp \left(j \Theta_{c}\right) \\
& =\boldsymbol{A}_{c}[w] \exp \left(j\left(\theta_{c}[w]+\Theta_{c}\right)\right)
\end{aligned}
$$

In (21), it can be seen that subcarriers that belong to the same cluster index $c$ are phase modulated with the same phase value. After modulating all the clusters, the transmitted signal is formed by applying an IFFT and then adding $\mathrm{CP}$ samples as in standard OFDM. Figure 3 shows a block diagram representation of the transmitter/receiver in the modified pilot-assisted baseband SLMOFDM architecture based on the ECM technique.

\subsection{Receiver}

As before, after the FFT stage at the receiver, each received subcarrier symbol in any given ECM modulated cluster may be expressed as

$$
\begin{aligned}
\overline{\boldsymbol{Z}}_{c}[w] & =\boldsymbol{H}_{c}[w] \boldsymbol{E}_{c}[w]+\boldsymbol{V}_{c}[w] \\
& =\boldsymbol{H}_{c}[w] \boldsymbol{X}_{c}[w] \exp \left(j \Theta_{c}\right)+\boldsymbol{V}_{c}[w] .
\end{aligned}
$$

First, sub-channel pilots components $\overline{\boldsymbol{H}}_{c}\left[w_{e}\right]$ and $\overline{\boldsymbol{H}}_{c}\left[w_{o}\right]$ are computed from

$$
\begin{aligned}
\overline{\boldsymbol{H}}_{c}\left[w_{e}\right] & =\overline{\boldsymbol{Z}}_{c}\left[w_{e}\right] / \boldsymbol{X}_{c}\left[w_{e}\right] \\
& =\left(\boldsymbol{H}_{c}\left[w_{e}\right] \boldsymbol{X}_{c}\left[w_{e}\right] \exp \left(j \Theta_{c}\right)+\boldsymbol{V}_{c}\left[w_{e}\right]\right) / \boldsymbol{X}_{c}\left[w_{e}\right] \\
& =\boldsymbol{H}_{c}\left[w_{e}\right] \exp \left(j \Theta_{c}\right)+\left(\boldsymbol{V}_{c}\left[w_{e}\right] / \boldsymbol{X}_{c}\left[w_{e}\right]\right) \\
& =\boldsymbol{H}_{c}\left[w_{e}\right] \exp \left(j \Theta_{c}\right)+\tilde{\boldsymbol{V}}_{c}\left[w_{e}\right]
\end{aligned}
$$


Transmitter

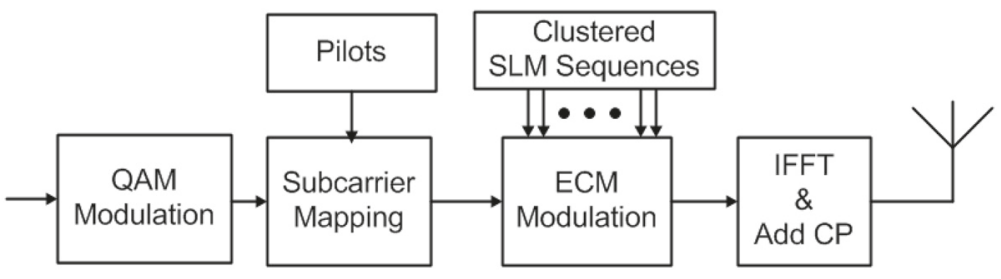

Receiver

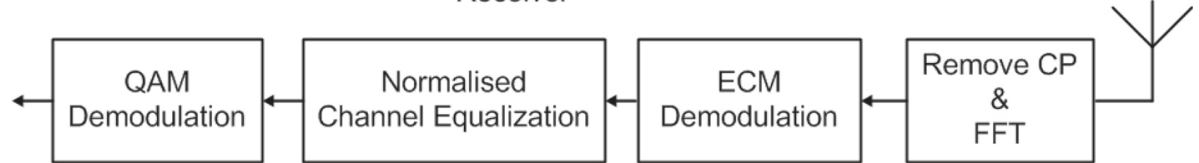

Fig. 3 A block diagram representation of a modified SLM-OFDM architecture based on the ECM technique

where $\tilde{\boldsymbol{V}}_{c}\left[w_{e}\right]=\left(\boldsymbol{V}_{c}\left[w_{e}\right] / \boldsymbol{X}_{c}\left[w_{e}\right]\right)$.

$$
\begin{aligned}
\overline{\boldsymbol{H}}_{c}\left[w_{o}\right] & =\overline{\boldsymbol{Z}}_{c}\left[w_{o}\right] / \boldsymbol{X}_{c}\left[w_{o}\right] \\
& =\left(\boldsymbol{H}_{c}\left[w_{o}\right] \boldsymbol{X}_{c}\left[w_{o}\right] \exp \left(j \Theta_{c}\right)+\boldsymbol{V}_{c}\left[w_{o}\right]\right) / \boldsymbol{X}_{c}\left[w_{o}\right] \\
& =\boldsymbol{H}_{c}\left[w_{o}\right] \exp \left(j \Theta_{c}\right)+\left(\boldsymbol{V}_{c}\left[w_{o}\right] / \boldsymbol{X}_{c}\left[w_{o}\right]\right) \\
& =\boldsymbol{H}_{c}\left[w_{o}\right] \exp \left(j \Theta_{c}\right)+\tilde{\boldsymbol{V}}_{c}\left[w_{o}\right]
\end{aligned}
$$

where $\tilde{\boldsymbol{V}}_{c}\left[w_{o}\right]=\left(\boldsymbol{V}_{c}\left[w_{o}\right] / \boldsymbol{X}_{c}\left[w_{o}\right]\right)$.

As before, in high SNR conditions, the expressions for $\overline{\boldsymbol{H}}_{c}\left[w_{e}\right]$ and $\overline{\boldsymbol{H}}_{c}\left[w_{o}\right]$ are reduced to

$$
\begin{aligned}
\overline{\boldsymbol{H}}_{c}\left[w_{e}\right] & \approx \boldsymbol{H}_{c}\left[w_{e}\right] \exp \left(j \Theta_{c}\right) \\
\overline{\boldsymbol{H}}_{c}\left[w_{o}\right] & \approx \boldsymbol{H}_{c}\left[w_{o}\right] \exp \left(j \Theta_{c}\right) .
\end{aligned}
$$

\subsubsection{ECM demodulation and SI cancellation}

Due to the use of the clustered SLM sequences defined in (19) and with respect to one of the pilot terms (e.g. $\left.\overline{\boldsymbol{H}}_{c}\left[w_{e}\right]\right)$, the cluster-based ECM demodulation procedure produces $\boldsymbol{Z}_{c}[w]$ through

$$
\begin{aligned}
\boldsymbol{Z}_{c}\left[w_{d}\right] & =\overline{\boldsymbol{Z}}_{c}\left[w_{d}\right] / \overline{\boldsymbol{H}}_{c}\left[w_{e}\right] \\
& =\frac{\left(\boldsymbol{H}_{c}\left[w_{d}\right] \boldsymbol{X}_{c}\left[w_{d}\right] \exp \left(j \Theta_{c}\right)+\boldsymbol{V}_{c}\left[w_{d}\right]\right)}{\boldsymbol{H}_{c}\left[w_{e}\right] \exp \left(j \Theta_{c}\right)+\tilde{\boldsymbol{V}}_{c}\left[w_{e}\right]} .
\end{aligned}
$$

Similarly, by omitting the additive noise components for simplicity and since they have a negligible effect at high SNR, the expression for $Z_{c}\left[w_{d}\right]$ is reduced to

$$
\begin{aligned}
\boldsymbol{Z}_{c}\left[w_{d}\right] & \approx \frac{\boldsymbol{H}_{c}\left[w_{d}\right]}{\boldsymbol{H}_{c}\left[w_{e}\right]} \boldsymbol{X}_{c}\left[w_{d}\right] \\
& \approx \boldsymbol{R}_{c}\left[w_{d}\right] \boldsymbol{X}_{c}\left[w_{d}\right]
\end{aligned}
$$

where $\boldsymbol{R}_{c}\left[w_{d}\right] \approx \boldsymbol{H}_{c}\left[w_{d}\right] / \boldsymbol{H}_{c}\left[w_{e}\right]$.
From the expression in (26), the variable $\boldsymbol{R}_{c}\left[w_{d}\right]$ is regarded as a normalised data sub-channel term (with respect to the $w_{e}$ indexed pilot in each cluster). Thus, similar to (25), the value of the equivalent normalised sub-channel for $w_{e}$ indexed pilot $\boldsymbol{R}_{c}\left[w_{e}\right]$ is always 1 since

$$
\boldsymbol{R}_{c}\left[w_{e}\right]=\overline{\boldsymbol{H}}_{c}\left[w_{e}\right] / \overline{\boldsymbol{H}}_{c}\left[w_{e}\right]=1 .
$$

Similarly, an equivalent $\boldsymbol{R}_{c}\left[w_{o}\right]$ is obtained from

$$
\begin{aligned}
\boldsymbol{R}_{c}\left[w_{o}\right] & =\overline{\boldsymbol{H}}_{c}\left[w_{o}\right] / \overline{\boldsymbol{H}}_{c}\left[w_{e}\right] \\
& =\frac{\left(\boldsymbol{H}_{c}\left[w_{o}\right] \exp \left(j \Theta_{c}\right)+\tilde{\boldsymbol{V}}_{c}\left[w_{o}\right]\right)}{\left(\boldsymbol{H}_{c}\left[w_{e}\right] \exp \left(j \Theta_{c}\right)+\tilde{\boldsymbol{V}}_{c}\left[w_{e}\right]\right)} .
\end{aligned}
$$

Again, by ignoring the additive noise terms in (27) at high SNR, the expression for $\boldsymbol{R}_{c}\left[w_{o}\right]$ is reduced to

$$
\boldsymbol{R}_{c}\left[w_{o}\right] \approx \boldsymbol{H}_{c}\left[w_{o}\right] / \boldsymbol{H}_{c}\left[w_{e}\right]
$$

The inherent SI cancellation features of the ECM technique is evident from the expressions in (25) to (28) since $\Theta_{c}$ is common to both numerator and denominator terms. This also suggests that since the knowledge of $\Theta_{c}$ is not required at the receiver, then the same ECM demodulation procedure can be applied when $\Theta_{c}$ is zero (without SLM) or non zero (with the clustered SLM). As a consequence, no SI estimation is required at the receiver.

\subsubsection{Normalised channel mitigation}

In contrast to the standard channel estimation highlighted in Section 2.2, a normalised channel estimation is used with ECM to mitigate the effect of the sub-channel term $\boldsymbol{R}_{c}\left[w_{d}\right]$ in (26). Using, for example, a linear interpolation between the normalised $w_{e}$ and $w_{o}$ indexed sub-channel terms $\boldsymbol{R}_{c}\left[w_{e}\right]$ and $\boldsymbol{R}_{c}\left[w_{o}\right]$, a normalised data sub-channel estimate $\hat{\boldsymbol{R}}_{c}\left[w_{d}\right]$ is obtained. 
Using the normalised channel estimate $\hat{\boldsymbol{R}}_{c}\left[w_{d}\right]$ and the representation of $\boldsymbol{Z}_{c}\left[w_{d}\right]$ in (26), a channel equalised term $\hat{Z}_{c}\left[w_{d}\right]$ is computed from $Z_{c}\left[w_{d}\right]$ through

$$
\begin{aligned}
\hat{\boldsymbol{Z}}_{c}\left[w_{d}\right] & =\boldsymbol{Z}_{c}\left[w_{d}\right] / \hat{\boldsymbol{R}}_{c}\left[w_{d}\right] \\
& \approx\left(\boldsymbol{R}_{c}\left[w_{d}\right] \boldsymbol{X}_{c}\left[w_{d}\right]\right) / \hat{\boldsymbol{R}}_{c}\left[w_{d}\right] .
\end{aligned}
$$

As before, an estimate of the transmitted data subcarrier symbol is obtained from $\hat{Z}_{c}\left[w_{d}\right]$ similar to the expression in (18).

\section{Simulation results}

Simulations were carried out to evaluate and compare the PAPR reduction capability and BER performance in a fading channel between the conventional SLM-OFDM system and the ECM method. Simulations also evaluate and compare the computational complexity between ECM data decoding procedures and the conventional SLMOFDM receiver that uses the FDC-based SI estimation scheme.

\subsection{CCDF performance}

The performance of PAPR reduction for both OFDM and ECM is evaluated using the well-known complementary cumulative distribution function (CCDF), which gives the probability of a PAPR value exceeding a certain threshold level $\gamma$ [4]. In this paper, 64-QAM data modulation is considered, as an example, in order to evaluate and compare the CCDF performance between conventional SLM and the modified SLM used in ECM.

The CCDF is computed as a function of $L$ and $N$. For CCDF evaluations, an oversampling factor of 4 is used as suggested in [19]. To demonstrate the effects of the cluster

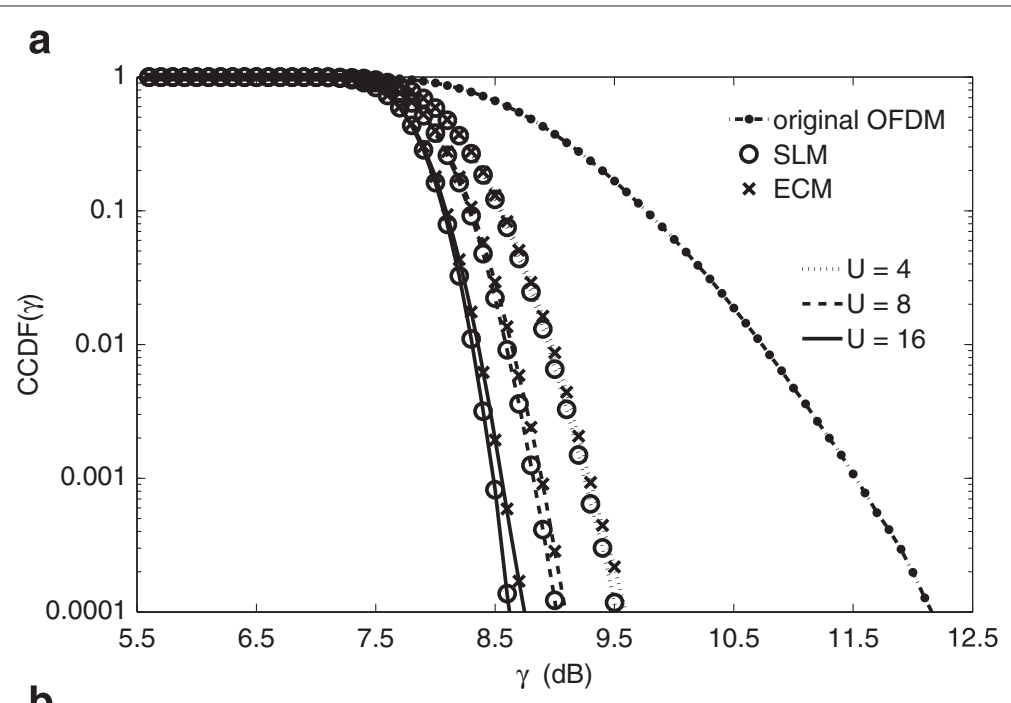

b

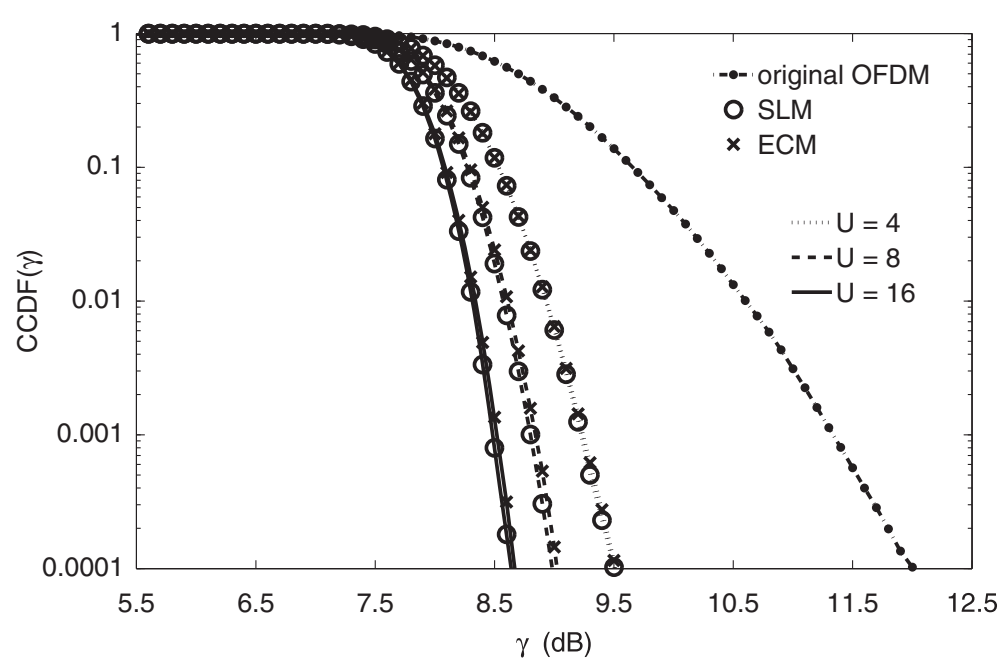

Fig. 4 CCDF comparisons $\left(N_{V}=128, N=512\right)$. $\mathbf{a} L=4$ and $\mathbf{b} L=8$ 


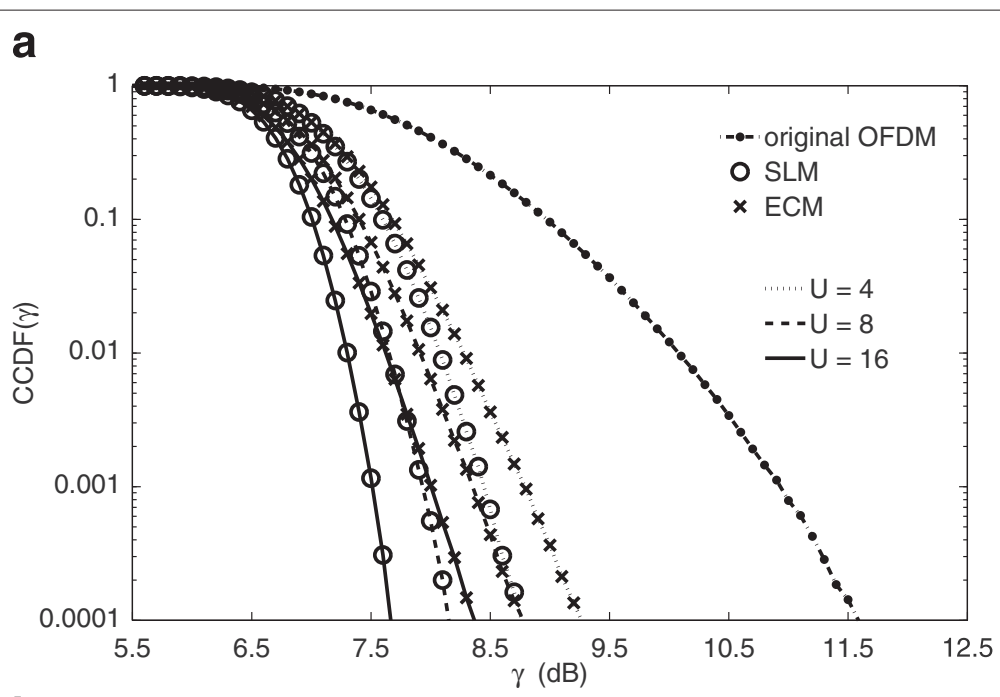

b

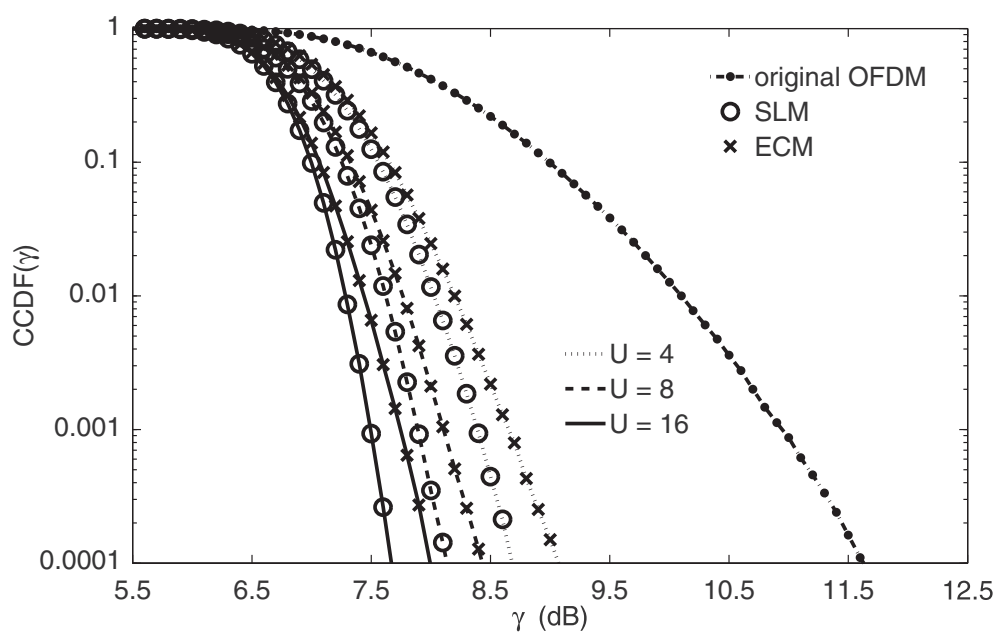

Fig. 5 CCDF comparisons ( $\left.N_{v}=512, N=2048\right)$. $\mathbf{a} L=4$ and $\mathbf{b} L=8$

size, $W$ on the CCDF evaluations, the pilot spacing $L$ is set to 4 and 8 . Note that $W=2 L$. In both conventional SLM and the modified SLM (in ECM), binary sequences, which consists of +1 and -1 are applied in a similar manner to $[8]$.
With $N_{v}=128$, Fig. 4a, b shows the CCDF comparisons with $L$ set to 4 and 8 , respectively. Fig. 5a, b shows similar results for a larger number of subcarriers where $N_{v}=512$. Results show that as expected, the PAPR reduction gain of conventional SLM is independent of the cluster

Table 1 Computational complexity of conventional data decoding with FDC SI estimation and ECM-based method

\begin{tabular}{|c|c|}
\hline Conventional data decoding (with FDC SI estimation) & ECM method \\
\hline $\begin{array}{l}\text { The FDC-based SI estimation method requires } 2 U N_{p}- \\
U \text { CMs and } U\left(N_{p}-2\right) \text { CAs to compute expressions in } \\
\text { (11) and (12) }\end{array}$ & $\begin{array}{l}\text { ECM requires } N_{p} \text { CMs to compute } \overline{\boldsymbol{H}}_{C}\left[w_{e}\right] \text { and } \overline{\boldsymbol{H}}_{C}\left[w_{0}\right] \\
\text { in (23) }\end{array}$ \\
\hline $\begin{array}{l}\text { SLM de-mapping requires } N_{V} \text { CMs to compute } \boldsymbol{Y}_{C}[w] \\
\text { in (13) }\end{array}$ & $\begin{array}{l}\text { The ECM demodulation procedure requires } N_{d} \text { CMs to } \\
\text { compute } \boldsymbol{Z}_{C}\left[w_{d}\right] \text { in }(25) \text { and } N_{p} / 2 \text { CMs to compute } \\
\boldsymbol{R}_{C}\left[w_{0}\right] \text { in }(27)\end{array}$ \\
\hline $\begin{array}{l}\text { Standard channel estimation and equalisation } \\
\text { requires } N_{V} \text { CMs to compute } \hat{\boldsymbol{H}}_{C}\left[w_{e}\right], \hat{\boldsymbol{H}}_{C}\left[w_{O}\right] \text { and } \\
\hat{\boldsymbol{Y}}_{C}\left[w_{d}\right]\end{array}$ & $\begin{array}{l}\text { The normalised channel equalisation procedure } \\
\text { requires } N_{d} \text { CMs to compute } \hat{\boldsymbol{Z}}_{C}\left[w_{d}\right] \text { in (30) }\end{array}$ \\
\hline
\end{tabular}


size. Results also show that the ECM method produces slightly less PAPR reduction performance compared with conventional SLM, particularly when the OFDM signal consists of a smaller number of subcarriers. With $N_{v}=128$, results in Fig. $4 \mathrm{a}, \mathrm{b}$ indicate that the PAPR reduction gain of the clustered SLM (i.e. ECM) is less when $L=8$ than for when $L=4$. For instance, when $U=8$ and with $N_{v}=128$, the conventional SLM achieves $\approx 0.3$ and $0.6 \mathrm{~dB}$ more PAPR reduction gain when $L$ is set to 4 and 8, respectively, compared with the ECM method. However, with $N_{v}=512$, both methods achieve almost identical PAPR reduction gain. Results therefore suggest that it is expected that the ECM-based PAPR reduction approach will produce slightly less PAPR reduction performance when the cluster size is large and $N_{v}$ is small.

The ECM method produces slightly less PAPR reduction gain because unlike conventional SLM, it uses the same phase shift for $W$ contiguous set of subcarriers in each cluster. However, for a larger number of subcarriers, the ECM method produces nearly similar PAPR reduction performance to conventional SLM, even with a larger cluster size of 16, i.e. when $L=8$.

\subsection{Computational complexity}

The computational complexity is now evaluated to further demonstrate an additional benefit of the ECM data decoding approach over a conventional SLM-OFDM receiver that uses, for example, the FDC-based SI estimation. This evaluation is based on the number of basic mathematical operations such as CMs and CAs. For the sake of simplicity, these evaluations ignore the computational complexity of channel interpolation and standard QAM demodulation in (18) because they are common to all considered methods.

Table 1 shows comparisons of computational requirements of the ECM method and conventional SLM-OFDM receiver that performs the FDC-based SI estimation. From Table 1, it can be seen that without taking the computational complexity of SI estimation into account, the SLM de-mapping, the channel estimation and the channel

Table 2 CCRR, $N_{v}=180, N_{p}=30$

\begin{tabular}{ll}
\hline$U$ & CCRR \\
\hline$U=2$ & $27 \%$ \\
$U=4$ & $42 \%$ \\
$U=8$ & $58 \%$ \\
$U=16$ & $73 \%$ \\
$U=32$ & $84 \%$ \\
$U=64$ & $91 \%$ \\
\hline
\end{tabular}

Table 3 Power-delay profile of the fading channel [23]

\begin{tabular}{lll}
\hline Taps & Path delays $(\mathrm{ns})$ & Average power $(\mathrm{dB})$ \\
\hline 1 & 0 & 0 \\
2 & 50 & -9.4 \\
3 & 100 & -18.9 \\
\hline
\end{tabular}

equalisation, then the procedures in conventional SLMOFDM receivers require a total of $2 N_{\nu}$ CMs. Similarly, the ECM-based data decoding procedures require a combined total of $2 N_{d}+N_{p}+N_{p} / 2 \mathrm{CMs} \approx N_{v}+N_{d}+N_{p} / 2$ CMs since $N_{v}=N_{p}+N_{d}$. Using the well-known computational complexity reduction ratio (CCRR) metric, the computational advantage of the ECM-based receiver is numerically evaluated relative to the standard SLM-OFDM receiver that performs FDC-based SI estimation. The CCRR is defined by [12]

$$
\mathrm{CCRR}=\left(1-\frac{\mathcal{C}_{\mathrm{ECM}}}{\mathcal{C}_{\text {std. }}}\right) \times 100 \%
$$

where $\mathcal{C}_{\mathrm{ECM}}$ and $\mathcal{C}_{\text {std. }}$. represent the number of $\mathrm{CM}$ operations required by the ECM- and FDC-based receiver, respectively. Note that the ECM technique requires no CAs.

Table 2 shows the evaluated CCRR as a function of $U$. Results in Table 2 show that the ECM method offers a reduction (in computational complexity) of about 27, 42 and $58 \%$ when $U$ is set to 2,4 and 8 , respectively. Therefore, the ECM approach offers a simplified data decoding procedure since it completely eliminates the computational complexity of SI estimation.

\subsection{BER performance}

Data recovery performance of conventional SLM-OFDM (with perfect SI estimation and with FDC based SI estimation) and ECM is evaluated when transmission is over an indoor residential frequency-selective fading channel characterised by the power-delay profile in Table 3 as defined by the joint technical committee (JTC) for wire-

Table 4 Simulation parameter values for BER evaluations

\begin{tabular}{ll}
\hline Parameters & Values \\
\hline$N, N_{V}, L$ & $256,180,6$ \\
$U$ & 8 \\
HPA model & Rapps model, $\rho=3[24]$ \\
IBO & $6 \mathrm{~dB}[13]$ \\
Data modulation & $16-$ QAM and 64-QAM \\
Channel fading & Rayleigh \\
\hline
\end{tabular}


less access [23]. The channel has a root mean square delay spread of $18 \mathrm{~ns}$. Simulations use parameter values outlined in Table 4.
To demonstrate the effects of HPA non-linearity on the BER performance, simulations use the well-known Rapps Model [24] to characterise HPA distortion. For an

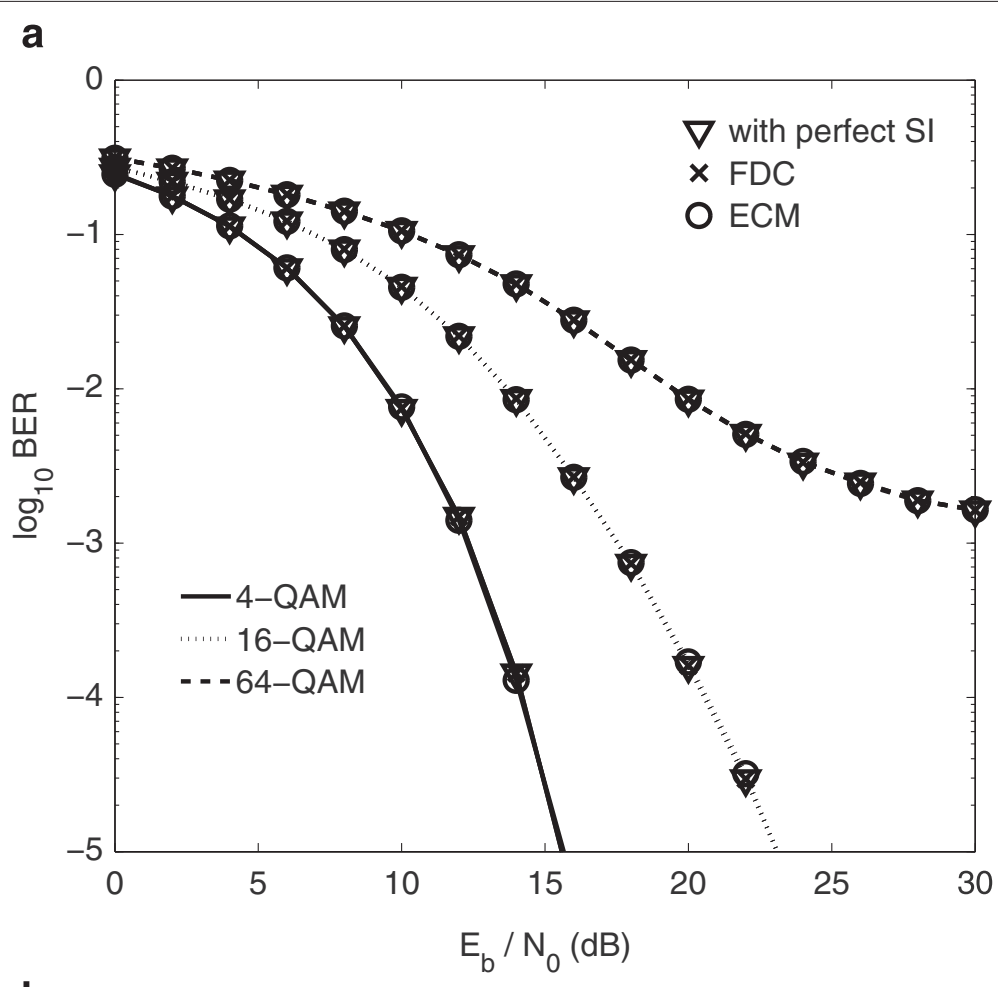

b

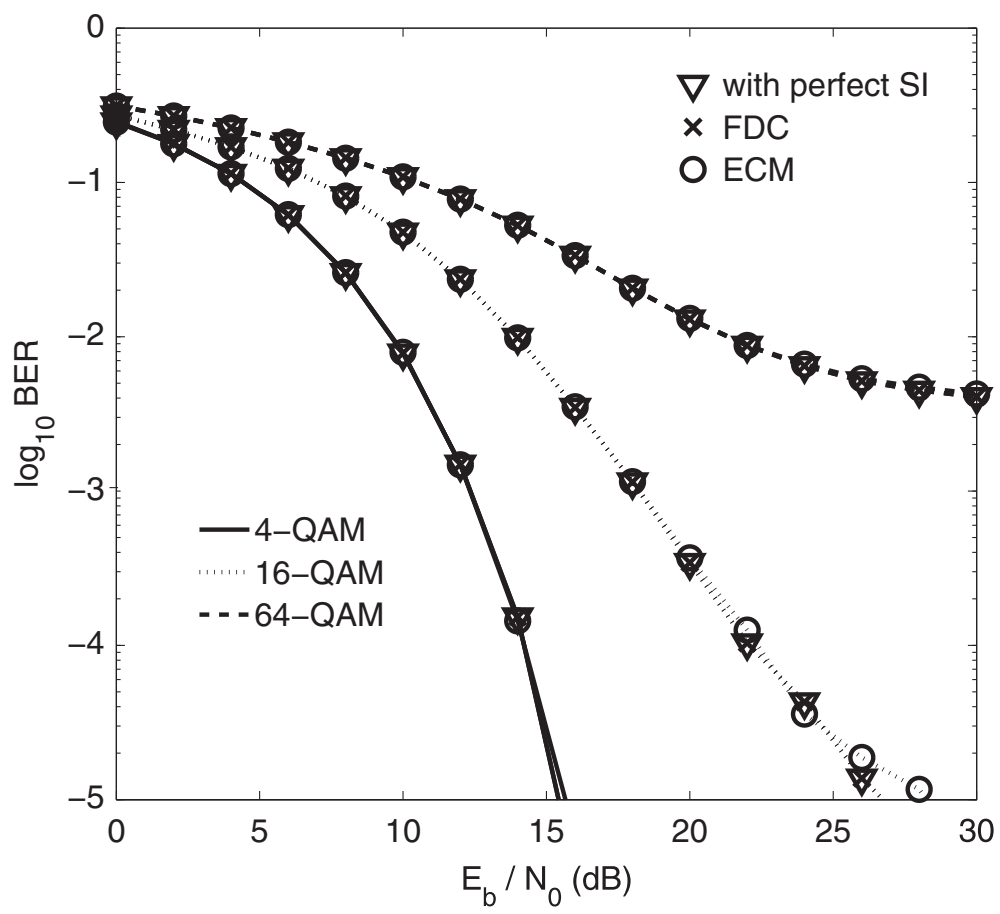

Fig. $6 \mathrm{BER}$ comparisons with and without amplifier distortions. a $\mathrm{NO} H P A, I B O=\infty$ and $\mathbf{b}$ with $\mathrm{HPA}, \mathrm{IBO}=6 \mathrm{~dB}$ 
arbitrary input signal, $\boldsymbol{x}(n)$, the output signal, $\boldsymbol{y}(n)$ from an HPA characterised by Rapp's model is obtained as [24]

$$
y(n)=\frac{x(n)}{\left[1+\left(\frac{|x(n)|}{A_{\text {sat }}}\right)^{2 \rho}\right]^{1 / 2 \rho}}
$$

where $A_{\text {sat }}$ is the amplifier's output saturation magnitude and $\rho$ is the smoothing factor which controls the HPA's transition from linear to saturation region, i.e. the higher the value of $\rho$, the sharper the transition from linear to non-linear operating region of the amplifier.

The linear region of an HPA is characterised by its IBO parameter, expressed by [16]

$$
\mathrm{IBO}(\mathrm{dB})=10 \log _{10}\left(\frac{P_{\text {sat }}}{P_{\text {avg }}}\right)
$$

where $P_{\text {sat }}$ and $P_{\text {avg }}$ denote the input saturation power and mean power of the input signal, respectively.

With no HPA distortions (i.e. IBO $=\infty$ ), Fig. 6a shows the BER comparisons between all considered methods. Using similar IBO level as in [13], Fig. 6b shows similar results in the presence of non-linear amplifier distortion with similar IBO value as that in [13] where the IBO value was 6 dB. Results in Fig. 6a, b show that all the considered methods produce identical BER performance even though no SI estimation is performed in the case of the ECM technique. As a consequence, the ECM method may be considered as an effective and alternative method to conventional SLM-OFDM, with the benefit that it requires no SI transmission or SI detection.

Though not presented here, similar results can be obtained for different cluster sizes. For a fixed value of $N_{v}$ and with a larger number of pilots (due to smaller pilot spacing), an improved BER can be obtained since a larger number of pilots can improve channel estimation [25]. Similarly, for the same $N_{v}$, a poorer BER performance is expected for a much larger pilot spacing.

\section{Conclusions}

This paper investigated the PAPR reduction and data decoding performances of a cluster-based modulation and demodulation method called ECM. The ECM method applies a slightly modified SLM to produce low PAPR signals. The PAPR reduction performance of the ECM method is dependent on the cluster size and the number of subcarriers. When compared with SLM, the ECM method achieves less PAPR reduction gain, particularly when the number of subcarriers is small. However, with a larger number of subcarriers, both methods produced almost identical PAPR reduction performance. At the receiver, the use of ECM-enabled data decoding with no need for SI estimation and as a result, eliminate the computational complexity of SI estimation. Results showed that the ECM method produced identical BER performance as other methods including when there exists perfect SI estimation and even in the presence of amplifier distortions.

To further improve the PAPR reduction performance of the ECM method, it is possible to increase the value of the SLM parameter $U$ since the computational complexity of ECM-based data decoding procedures is independent of the value of $U$.

\section{Abbreviations}

AWGN, additive white Gaussian noise; BER, bit error rate; CA, complex addition; CCDF, complementary cumulative distribution function; $C M$, complex multiplication; CP, cyclic prefix; ECM, embedded coded modulation; FDC, frequency-domain correlation; FFT, fast Fourier transform; HPA, high power amplifier; IBO, input back-off; IFFT, inverse fast Fourier transform; JTC, joint technical committee; LTE, long-term evolution; OFDM, orthogonal frequency division multiplexing; PAPR, peak-to-average power ratio; PASIE, pilot-assisted side information estimation; PTS, partial transmit sequence; SI, side information; SLM, selected mapping; SNR, signal-to-noise ratio; QAM, quadrature amplitude modulation

\section{Competing interests}

The authors declare that they have no competing interests.

Received: 25 November 2015 Accepted: 3 April 2016

Published online: 18 April 2016

\section{References}

1. K Bae, JG Andrews, EJ Powers, Quantifying an iterative clipping and filtering technique for reducing PAR in OFDM. Wireless Commun. IEEE Trans. 9(5), 1558-1563 (2010)

2. THwang, C Yang, G Wu, S Li, GY Li, OFDM and its wireless applications: a survey. Vehic. Technol. IEEE Trans. 58(4), 1673-1694 (2009)

3. K Bae, C Shin, EJ Powers, Performance analysis of OFDM systems with selected mapping in the presence of nonlinearity. Wireless Commun. IEEE Trans. 12(5), 2314-2322 (2013)

4. T Jiang, Y Wu, An overview: peak-to-average power ratio reduction techniques for OFDM signals. Broadcasting, IEEE Trans. 54(2), 257-268 (2008)

5. Y Rahmatallah, S Mohan, Peak-to-average power ratio reduction in OFDM systems: a survey and taxonomy. Commun. Surveys Tutor. IEEE. 15(4), 1567-1592 (2013)

6. D-W Lim, S-J Heo, J-S No, An overview of peak-to-average power ratio reduction schemes for OFDM signals. Commun. Netw. J. 11(3), 229-239 (2009)

7. RW Bauml, RFH Fischer, JB Huber, Reducing the peak-to-average power ratio of multicarrier modulation by selected mapping. Electron. Lett. 32(22), 2056-2057 (1996)

8. GT Zhou, L Peng, Optimality condition for selected mapping in OFDM. Signal Process. IEEE Trans. 54(8), 3159-3165 (2006)

9. RJ Baxley, GT Zhou, Comparing selected mapping and partial transmit sequence for PAR reduction. Broadcast. IEEE Trans. 53(4), 797-803 (2007)

10. ADS Jayalath, C Tellambura, SLM and PTS peak-power reduction of OFDM signals without side information. Wireless Commun. IEEE Trans. 4(5), 2006-2013 (2005)

11. SY Le Goff, SS Al-Samahi, BK Khoo, CC Tsimenidis, BS Sharif, Selected mapping without side information for PAPR reduction in OFDM. Wireless Commun. IEEE Trans. 8(7), 3320-3325 (2009)

12. J Park, E Hong, D Har, Low complexity data decoding for SLM-based OFDM systems without side information. Commun. Lett. IEEE. 15(6), 611-613 (2011)

13. E Hong, H Kim, K Yang, D Har, Pilot-aided side information detection in SLM-based OFDM systems. Wireless Commun. IEEE Trans. 12(7), 3140-3147 (2013)

14. J Ji, G Ren, H Zhang, A semi-blind SLM scheme for PAPR reduction in OFDM systems with low-complexity transceiver. Vehicular Technol. IEEE Trans. 64(6), 2698-2703 (2015) 
15. ETSI European Standard EN 302755 v1.3.1, Digital Video Broadcasting (DVB); Frame structure channel coding and modulation for a second generation digital terrestrial television broadcasting system (DVB-T2), 2011

16. SA Adegbite, S McMeekin, BG Stewart, Performance of a new joint PAPR reduction and SI estimation technique for pilot-assisted SLM-OFDM systems, Communication Systems, Networks \& Digital Signal Processing (CSNDSP), 2014 9th International Symposium On, Manchester, 2014, 308-313

17. SD Stearns, DR Hush, Digital signal processing with examples in MATLAB, CRC press. 2002

18. A Peled, A Ruiz, Frequency domain data transmission using reduced computational complexity algorithms, Acoustics, Speech, and Signal Processing, IEEE International Conference on ICASSP' $80 ., 1980,964-967$

19. T Jiang, M Guizani, H-H Chen, W Xiang, Y Wu, Derivation of PAPR distribution for OFDM wireless systems based on extreme value theory. Wireless Commun. IEEE Trans. 7(4), 1298-1305 (2008)

20. WO Popoola, Z Ghassemlooy, BG Stewart, Pilot-assisted PAPR reduction technique for optical OFDM communication systems. Lightwave Technol. J. 32(7), 1374-1382 (2014)

21. SA Adegbite, SG McMeekin, BG Stewart, Low-complexity data decoding using binary phase detection in SLM-OFDM systems. Electron. Lett. 50(7), 560-562 (2014)

22. S Coleri, M Ergen, A Puri, A Bahai, Channel estimation techniques based on pilot arrangement in OFDM systems. Broadcasting, IEEE Trans. 48(3), 223-229 (2002)

23. Joint Technical Committee on Wireless Access, Final report on RF channel characterization, 1993

24. C Rapp, in Proc. 2nd European Conference on Satellite Communication. Effects of HPA nonlinearity on a 4-DPSK/OFDM signal for a digital sound broadcasting system, (Liege, Belgium, 1991), pp. 179-184

25. MK Ozdemir, H Arslan, Channel estimation for wireless OFDM systems. Commun. Surv. Tutor. IEEE. 9(2), 18-48 (2007)

\section{Submit your manuscript to a SpringerOpen ${ }^{\circ}$ journal and benefit from:}

- Convenient online submission

- Rigorous peer review

- Immediate publication on acceptance

- Open access: articles freely available online

- High visibility within the field

- Retaining the copyright to your article

Submit your next manuscript at $\gg$ springeropen.com 